\title{
Depression in individuals with spinal cord injury: methodological issues
}

\author{
$\mathrm{KS} \mathrm{Jacob}^{1}, \mathrm{~K}$ Zachariah ${ }^{2}$ and S Bhattacharji ${ }^{2}$ \\ ${ }^{1}$ Department of Psychiatry, Christian Medical College, Vellore 632002; ${ }^{2}$ Department of Physical Medicine and \\ Rehabilitation, Christian Medical College and Hospital, Vellore 632004, India
}

The methodological problems in research related to depression in individuals with spinal cord injury (SCI) are examined. These include relation to normal emotional reactions following injury, the use of physical and vegetative symptoms in the diagnosis of depression, the utilisation of rating scales primarily designed for use in psychiatric populations, the heterogeneity of patients with SCI, the role of preexisting psychiatric morbidity, the selection of controls for comparison and the necessity for multivariate statistical approaches to analysis. The issues and possible solutions are discussed.

Keywords: spinal cord injury; depression; methodological studies; psychological reactions

\section{Introduction}

Psychiatric morbidity has been reported in individuals with various types of medical (physical) illness. ${ }^{1-3}$ Several mental disorders and psychological problems have also been documented in patients with spinal cord injury (SCI).${ }^{4.5}$ Depression is one of the major psychological reactions described. ${ }^{6,7}$ However, most of the investigations studying this aspect of SCI have significant methodological limitations. Prior to the acceptance of the findings, the procedural difficulties have to be addressed. This paper attempts to discuss some of the issues involved.

\section{Methodological issues}

The issues are examined under the following headings:

(1) the diagnosis;

(2) the diagnostic criteria employed;

(3) the rating of severity;

(4) the heterogeneity of the population;

(5) psychiatric morbidity prior to SCI;

(6) other issues.

\section{The diagnosis}

The loss of physical function in SCI produces significant distress in psychologically healthy individuals. The emtional reactions following the development of the condition are similar to those recorded in bereavement. The psychological response and its resolution are said to be broadly comparable. While many workers accept a period of 'mourning' after becoming paraplegic or tetraplegic, ${ }^{6,8}$ others argue against such generalisation. ${ }^{9,10}$ The reason for this apparent contradiction is the absence of a universal response to stress in general and to SCI in particular. The intensity, duration and quality of grief after bereavement and after SCI are variable. From a conceptual point of view the need to accept the new reality (ie implications of bereavement or the disability after SCI) is mandatory for a healthy adjustment. We feel that this process of acceptance is regarded as the mourning/grief process. It is not inevitable that all individuals with SCI present with 'severe depression' and can be labelled as going through the grief process. But mourning after SCI is mandatory for healthy adaptation.

The presentations of normal grief following bereavement are difficult to differentiate from a clinical depressive syndrome as there is a marked overlap of symptoms. ${ }^{11-13}$ The normality of grief responses is determined more by sociocultural criteria than by biological standards. Consequently, individuals with normal grief reactions cannot be diagnosed as having a major depression/depressive episode by the current psychiatric classifications (eg Diagnostic and Statistical Manual III $^{14}$ and III Revised ${ }^{15}$ and the International Classification of Diseases $10^{16}$ ). However, despite the acceptance of the normalcy of psychological reactions seen in the majority of individuals with SCI, these are labelled abnormal by these diagnostic systems. Investigations employing such diagnostic standards would be, we say, inaccurate.

Abnormal psychological responses to $\mathrm{SCI}$ have to be based on criteria employed to assess pathology in bereavement. These include extreme severity of emotional turmoil, prolonged grief and qualitative changes suggestive of a psychotic process. ${ }^{11-13}$ These are general guidelines and demand interpretations based on sociocultural norms.

\section{Diagnostic criteria employed}

The past two decades have witnessed major advances in the diagnosis of various depressive disorders. ${ }^{14-16}$ The 
reliability and validity of diagnostic criteria for mental disorders have been examined. However, these definitions have been standardised for psychiatric populations. Their use in medical and non-psychiatric settings demands caution, as these patients differ significantly from those attending psychiatric facilities.

A major issue is the altered significance of physical and vegetative symptoms in the diagnosis of depression in individuals with medical disease. Criteria for depressive disorders employed in psychiatric settings tend to include physical (eg weakness, fatigue) and vegetative symptoms (eg sleep disturbance, loss of appetite and weight) for diagnosis. In medical patients and individuals with SCI these symptoms can be secondary to the underlying physical problems (eg pain, weakness). Distinguishing the cause of such symptoms with certainty is difficult. The direct application of such standards to patients with SCI would result in errors in diagnosis and investigations ${ }^{8,10}$ using these criteria are prone to inaccuracy.

The literature on attempts to overcome the problems posed by physical and vegetative symptoms can be classifed into four categories. ${ }^{2}$ Some workers have adopted an 'inclusive' strategy which counts all symptoms as due to depression (physical, vegetative or otherwise) whether or not they are secondary to the physical illness. ${ }^{2,8}$ The sensitivity of this approach is high but it results in lower specificity with an increase in the false positive rate. An alternative is the 'aetiological' method as described in the Diagnostic and Statistical Manual III Revised ${ }^{2,15}$ which suggests that the diagnostician counts symptoms only if they are not caused by physical illness. Such an approach requires inference and hence has low reliability. The 'substitutive' strategy substitutes such symptoms with others. ${ }^{2,6}$ However, its reliability and validity have not been evaluated. The fourth tactic, called 'exclusive', eliminates such symptoms resulting in increased specificity but low sensitivity with increased false negatives. ${ }^{2,6}$ The inclusive strategy has been recommended for clinical settings (to institute treatment) and the exclusive approach advocated for research (to identify the core group). The inclusive strategy when employed during the process of normal grief results in a large number of false positives.

Considering these problems, we feel that there is a definite need for a re-evaluation of the issues involved. Criteria have to be designed and specifically tested for their usefulness in patients with SCI. Ideal standards require to be evolved employing a 'substitutive' approach. Physical and vegetative symptoms will require to be replaced by psychological criteria. The reliability and validity of such standards will have to be established. However, until such time the currently available criteria are used (after excluding normal emotional reactions to $\mathrm{SCI}$ ) employing the 'inclusive' strategy for treatment and the 'exclusive' approach for research.

The rating of severity

Many scales have been devised to measure the severity of depression in psychiatric populations. These include the Beck Depression Inventory, ${ }^{17}$ Zung self rating depression scale, ${ }^{18}$ Hamilton rating scale for depression $-17,{ }^{19}$ Hamilton rating scale for depression$21,{ }^{20}$ Montgomery-Asberg depression rating scale, ${ }^{21}$ and melancholia scale. ${ }^{22}$ However, these instruments include somatic and vegetative symptoms and consequently are inappropriate for use in medical and SCI populations. $^{23}$ The majority of studies assessing depression in SCI have employed these instruments and their conclusions are therefore suspect.

The hospital anxiety and depression scale ${ }^{24}$ is the only instrument currently available which does not contain such symptoms. It was originally standardised in medically ill patients and found to have high validity and reliability. Consequently its use in SCI patients would be appropriate. The only disadvantage of this scale is that it is a self rated instrument and cannot be employed in semi-literate individuals and in those whose depression makes them indecisive. The measurement of depression in such patients would require the construction and standardisation of an observer rated scale.

A related issue is the use of severity ratings from depression rating scales for the diagnosis of the condition. Some studies have employed such a strategy without attempting to make a clinical diagnosis of depression. ${ }^{7,25,26}$ Such a strategy is inherently erroneous. Scales designed for the assessment of severity of depression are not diagnostic instruments and should not be used as such.

\section{Heterogeneity of population}

Many inquiries have studied SCI individuals with diverse physical problems producing a heterogeneous patient population. ${ }^{7,8,10,25,27}$ For example, the variable sites and the completeness of the lesion result in dissimilar handicap and mobility. From the patient's point of view it is the handicap in terms of function rather than the lesion per se which determine the extent of the psychological adjustments that are required. Individuals with paraplegia differ from those with tetraplegia and should not be grouped together. Mixed patient populations have diverse psychological problems which cannot be generalised to subgroups. Inquiries attempting to document psychological reactions have to examine the relation to the handicap.

\section{Psychiatric morbidity prior to SCI}

Psychiatric morbidity prior to the onset of paraplegia colours the subsequent psychological reactions. Individuals with major depression, schizophrenia and other mental disorders who develop SCI following unsuccessful suicide attempts would have their primary psychiatric pathology complicating the psychological process of recovery. Similarly, the stress of being a tetraplegic/paraplegic person can precipitate mental disorders in individuals with a past history of such conditions. Such patients would have to be studied separately from other individuals who develop SCI. 
Many studies assessing emotional problems in those with paraplegia do not separate individuals with 'preSCI' psychiatric morbidity. ${ }^{10,26}$ Inquiries would have to actively separate these two populations and examine issues independently.

\section{Other issues}

Many studies tend to compare individuals with SCI with matched healthy controls. ${ }^{7,9,28}$ Such inquiries tend to conclude that SCI is associated with increased psychological morbidity. While such a conclusion is true at a superficial level, the psychological problems documented may be non-specific reactions to increased stress in general rather than being distinctive to SCI. In order to prove that such reactions are characteristic of SCI, the controls selected should be from populations under a similar magnitude of stress (eg patients with other serious medical problems). Unless the controls are comparable regarding the type of stress it would be difficult to claim that the psychological sequelae of SCI are specific to that condition. Such studies may be futile in practice as it could be argued that the controls are not comparable. Studies on SCI patients should focus on the examining issues within SCI population (eg comparison of depressed versus non-depressed patients, risk factors associated with depression etc).

Many studies on depression in those who have SCI tend to examine risk factors (which are associated with course and outcome) within a univariate framework. ${ }^{29-32}$ However, the correlations among these variables are complex. For example, social supports ${ }^{33}$ or personality structure may modify the effect of SCI on the individual and would have to be accounted for. Thus, it is mandatory to employ multivariate statistical approaches to the analysis of such data. ${ }^{34}$

\section{Conclusions}

Although the importance of the recognition and management of depression and psychiatric morbidity in individuals with SCI has been recognised in the literature, the field is plagued with methodological difficulties. In fact these problems are a major reason for the contradictory findings with regard to prevalence, quality of emotional reactions, course and outcome of depression. The problems discussed also apply to depression and psychiatric morbidity in individuals with serious medical illness and to other psychological presentations noted in SCI. There is a definite need for a reappraisal of the issues involved and for more systematic research.

\section{Acknowledgement}

The authors would like to thank Dr PJ Saju for his comments on the earlier drafts of the manuscript.

\section{References}

1 Rodin G, Voshart K. Depression in the medically ill: an overview. Am J Psychiatry 1986; 143: 696-705.
2 Cohen-Cole SA, Stoudemire A. Major depression and physical illness: special considerations in diagnosis and biologic treatment. Psychiatr Clin North Am 1987; 10: 1-17.

3 Cassem EH. Depression and anxiety secondary to medical illness. Psychiatr Clin North Am 1990; 13: 597-612.

4 Judd FK, Burrows GD. Liaison psychiatry in a spinal injuries unit. Paraplegia 1986; 24: 6-19.

5 Judd FK, Brown DJ. Psychiatry in the spinal injuries unit. Paraplegia 1987; 25: 254-257.

6 Judd FK, Brown DJ, Burrows GD. Depression, disease and disability: application to patients with traumatic spinal cord injury. Paraplegia 1991; 29: 91-96.

7 Hancock KM et al. Anxiety and depression over the first year of spinal cord injury: a longitudinal study. Paraplegia 1993; 31: 349-357.

8 Judd FK et al. Depression following spinal cord injury: a prospective inpatient study. Br J Psychiatry 1989; 154: 668-671.

9 Richards JS. Psychologic adjustment to spinal cord injury during first postdischarge year. Arch Phys Med Rehabil 1986; 67: 362-365.

10 Fullerton DT, Harvey RF, Klein MH, Howell T. Psychiatric disorders in patients with spinal cord injuries. Arch Gen Psychiatry 1981; 38: 1369-1371.

11 Gonda TA. Death, dying and bereavement. In: Kaplan HI, Sadock BJ (eds). Comprehensive Textbook of Psychiatry, 5th edn. Williams and Wilkins: Baltimore, 1989, pp 1339-1351.

12 Clayton PJ. Bereavement. In: Payekl ES (ed). Handbook of Affective Disorders. Churchill Livingstone: Edinburgh, 1982.

13 Middleton W, Raphael B. Bereavement: state of the art and state of the science. Psychiatr Clin North Am 1987; 10: 329-344.

14 American Psychiatric Association. Diagnostic and Statistical Manual III. American Psychiatric Association: Washington, 1980.

15 American Psychiatric Association. Diagnostic and Statistical Manual III Revised. American Psychiatric Association: Washington, 1987.

16 World Health Organization. International Classification of Diseases. 10: Clinical descriptions and diagnostic guidelines. World Health Organization: Geneva, 1992.

17 Beck AT et al. An inventory for measuring depression. Arch Gen Physchiatry 1961; 4: 561-571.

18 Zung WWK. A self rating depression scale. Arch Gen Psychiatry $1965 ; 12$ : 63-70.

19 Hamilton M. A rating scale for depression. J Neurol Neurosurg Psychiatry 1960; 23: 56-62.

20 Hamilton M. Development of a rating scale for primary depressive illness. Br J Soc Clin Psychol 1967; 23: 56-62.

21 Montgomery SA, Asberg MA. A new depression scale designed to be sensitive to change. Br J Psychiatry 1979; 134: 382-389.

22 Bech P, Rafaelsen OJ. The use of rating scales exemplified by a comparison of the Hamilton and the Bech-Rafaelsen melancholia scale. Acta Psychiatr Scand 1980; 62 (Suppl 285): 128-131.

23 Snaith P. What do rating scales measure? Br J Psychiatry 1993; 163: 293-298.

24 Zigmond AS, Snaith RP. The hospital anxiety and depression scale (HAD). Acta Psychiatr Scand 1983; 67: 361-370.

25 Frank RG, Elliott TR. Spinal cord injury and health locus of control beliefs. Paraplegia 1989; 27: 250-256.

26 Somasundaram O, Balakrishnan S, Ravindran OS, Shanmugasundaram TK. Psychological study of spinal cord injured patients involved in the Madras Paraplegia Project. Paraplegia 1992; 30: 799-802.

27 Macleod AD. Self-neglect of spinal injured patients. Paraplegia 1988; 26: 340-349.

28 Fuhrer MJ et al. Depressive symptomatology in persons with SCI who reside in the community. Arch Phys Med Rehabil 1993; 74: 255-260.

29 Frank RG, Elliott TR. Life stress and psychological adjustment following spinal cord injury. Arch Phys Med Rehabil 1987; 68: 344-347.

30 Goldberg RT, Freed MM. Vocational development of spinal cord injury patients: an 8 year followup. Arch Phys Med Rehabil 1982; 63: 207-210.

31 Green BC, Pratt CC, Grigsby TE. Self concept among persons 
with long term spinal cord injury. Arch Phys Med Rehabil 1984; 65: $751-754$.

32 MacDonald MR, Neilson WR, Cameron MGP. Depression and activity patterns of spinal cord injured persons living in the community. Arch Phys Med Rehabil 1987; 68: 339-343.
33 Anson CA, Stanwyck DJ, Krause JS. Social support and health status in spinal cord injury. Paraplegia 1993; 31: 632-638.

34 Krause JS, Crewe NM. Long term prediction of self reported problems following spinal cord injury. Paraplegia 1990; 28: 186-202. 